\title{
Norois
}

Environnement, aménagement, société

$234 \mid 2015$

Dynamique des milieux humides, éducation à l'environnement, pratiques festives, tourisme et patrimonialisation

\section{Dinan en représentation(s). Patrimonialisation et mise en tourisme d'une petite ville historique de Bretagne}

Representations of Dinan. Heritage-making and development of tourism of a small historic city in Brittany

Annie Ouellet

\section{(2) OpenEdition}

Journals

Édition électronique

URL : https://journals.openedition.org/norois/5572

DOI : $10.4000 /$ norois. 5572

ISBN : 978-2-7535-4218-1

ISSN : $1760-8546$

Éditeur

Presses universitaires de Rennes

Édition imprimée

Date de publication : 30 juin 2015

Pagination : 47-63

ISBN : 978-2-7535-4183-2

ISSN : 0029-182X

Référence électronique

Annie Ouellet, «Dinan en représentation(s). Patrimonialisation et mise en tourisme d'une petite ville historique de Bretagne », Norois [En ligne], 234 | 2015, mis en ligne le 30 juin 2017, consulté le 14 janvier 2022. URL : http://journals.openedition.org/norois/5572 ; DOI : https://doi.org/10.4000/norois. 5572

(c) Tous droits réservés 


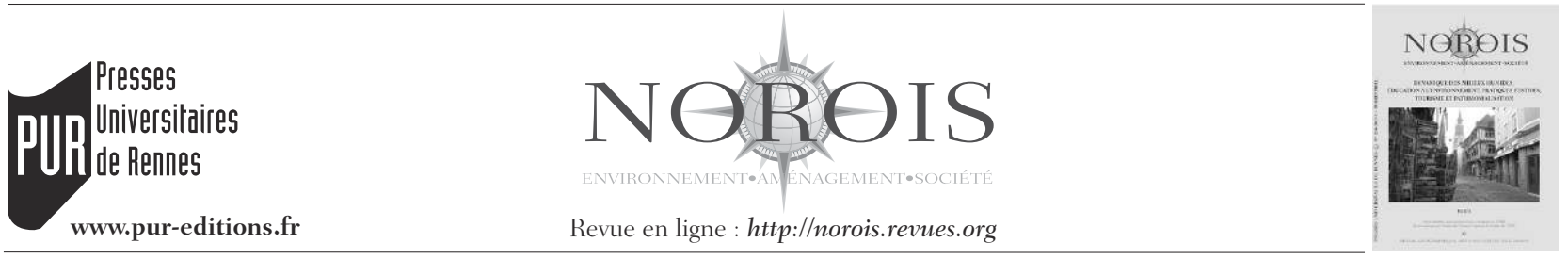

\title{
Dinan en représentation(s) Patrimonialisation et mise en tourisme d'une petite ville historique de Bretagne
}

\author{
Representations of Dinan \\ Heritage-making and Development of Tourism of a Small Historic City in Brittany
}

\author{
Annie Ouellet
}

UMR 6590 CNRS ESO, Université d'Angers, 5 bis boulevard Lavoisier - 49045 ANGERS - France (annieouellet22@

gmail.com)

Résumé : Cet article explore la question des représentations de Dinan, petite ville bretonne où le double-processus de patrimonialisation et de mise en tourisme est largement basé sur l'image de cité médiévale. Une approche méthodologique fondée sur l'outil photographique et croisée à des entretiens avec les deux principaux groupes se côtoyant au sein de cette ville, soit les résidents et les touristes, a été mise en place. Elle nous a permis de mieux comprendre la construction des représentations qu'ont d'une même ville, deux groupes s'y trouvant dans des contextes spatio-temporels distincts. Aussi, l'impact des mises en tourisme et en patrimoine sur ces représentations a pu être interrogé. Une convergence des regards des touristes et des résidents est apparue de façon marquée, de même qu'une certaine forme de rejet, de la part des résidents, des espaces considérés comme «trop » touristiques.

Abstract: This article explores the matter of the representations of Dinan, a small town in Brittany where the dual process of heritagemaking and development of tourism is mainly based on the image of a medieval city. We have used a methodological approach based on a photographic tool and crossed with interviews of both groups mixing in this town: the inhabitants and the tourists. This method has helped us to understand the way the representations of a same town were constructed by two groups evolving in distinct spatiotemporal contexts and the impact of the development of tourism and heritage-making on this construction. A "convergence" of the views has appeared and a rejection, by the inhabitants, of spaces considered as "too" touristic.

Mots clés : Patrimonialisation - mise en tourisme - représentations - petite ville historique - Dinan

Keywords: Heritage-Making - development of tourism - representation - small historic city - Dinan

\section{INTRODUCTION}

À une époque où le futur semble particulièrement incertain, se rappeler de ce qui a été prend la forme d'une injonction. Ce devoir de mémoire trouve ainsi fréquemment son accomplissement dans la conservation du patrimoine bâti, celui-ci permettant d'en préserver une expression tangible. Françoise Choay écrivait d'ailleurs à ce propos que le monument 
« rassure, tranquillise en conjurant l'être du temps » (Choay, 1992, p. 15). En ce sens, les villes portant encore les traces matérielles de ce passé et de l'identité des sociétés qui les ont habitées au fil des siècles, que l'on nommera ici les villes historiques, sont particulièrement valorisées. La volonté de ces dernières de mettre en valeur leur patrimoine ${ }^{1} \mathrm{va}$, dès lors, le plus souvent de pair avec la mise en tourisme, soit la promotion de la ville en tant que destination.

Aussi, pour répondre à cet objectif, une certaine esthétisation de la ville semble s'imposer. Bien que cette prégnance de l'image puisse être généralisable à l'ensemble de la société moderne (Boorstin, 1963 [1961]), elle est, dans le cas des villes historiques soumises à ce double processus de mise en tourisme et en patrimoine, poussée à l'excès. Mise en tourisme et mise en patrimoine se croiseraient, se superposeraient ainsi afin de créer une image patrimoniale touristique, la ville devenant un simple décor (Urry, 1990; Chazaud, 1994). On peut alors interroger le rôle du touriste dans ce processus. Considère-t-il la ville qu'il visite comme étant mise en scène ${ }^{2}$ ? N'est-il qu'un spectateur de cette représentation? Ne peut-il pas en être acteur en modifiant les images qui lui sont soumises, en construisant ses propres représentations?

Aussi, alors que certains auteurs se sont intéressés aux représentations des lieux du quotidien, en tant que paysages banals ou ordinaires (Luginbühl, 1989, 2012; Michelin, 1998; Lelli, 2000 ; Bigando, 2006, 2008), qu'en est-il lorsque ce lieu du quotidien est élevé, par un classement patrimonial ou encore par le regard de touristes, au rang de lieu de grande qualité? L'un des buts affichés de l'Association des Villes et Pays d'Art et d'Histoire (VPAH) est d'ailleurs de faire prendre conscience aux résidents de ces villes « qu'ils ne vivent pas dans une ville ordinaire, mais bien dans une ville extra-ordinaire ${ }^{3}$ ». Il est alors intéressant de questionner également les représentations qu'ont les habitants de leur ville, de voir comment elles se positionnent entre ces pôles.

1. La mise en valeur du patrimoine étant ici entendue selon le double-sens évoqué par Alain Roussillon (2010), qui serait à la fois de l'exhiber (le mettre en valeur), mais aussi d'en tirer profit.

2. Tel qu'évoqué par la Direction de l'Architecture et du Patrimoine comme un objectif des Villes et Pays d'Art et d'Histoire (cité par Irvoas-Dantec, 2003).

3. Entretien avec monsieur René Benoît (mars 2013), maire de Dinan (de 1983 à 2014) et président de l'Union des Villes d'Art et d'Histoire et des Villes Historiques de Bretagne.
Notre propos s'articule en quatre points mettant en exergue trois éléments structurants de notre travail. Nous présenterons les aspects théoriques et méthodologiques, ces derniers s'appuyant essentiellement sur une démarche qualitative, croisant l'entretien et la photographie, puis nous aborderons la spécificité de la petite ville historique comme cadre spatial d'analyse à travers l'exemple de Dinan. Finalement nous traiterons des représentations des touristes et des résidents. Même si elles sont traitées séparément, notre volonté est bien de mettre en perspectives les représentations de ces deux groupes.

\section{RÉVÉLER LES REPRÉSENTATIONS : ÉLÉMENTS THÉORIQUES ET MÉTHODOLOGIQUES}

«What makes a particular tourist gaze depends upon what it is contrasted with; what the forms of non-tourist experience happen to be. » (Urry, 1990, p. 2)

Suivant ces propos de John Urry, nous pourrions postuler que les représentations qu'ont les touristes et les résidents d'une même ville, sont fondamentalement différentes. Toutefois, une telle hypothèse admettrait que la "spectacle-isation of space ", expression du même auteur, n'affecterait que le « regard du touriste », alors que celui du résident, y serait complètement indifférent. Nous émettons quelques réserves par rapport à un tel constat. Nous postulons plutôt que cette distinction entre touristes et résidents n'est pas si nette et tranchée. Nous posons l'hypothèse que l'esthétisation de la ville touristique patrimoniale, élaborée par les élus, les institutions locales ou certains organismes de promotion touristique, bien que visant prioritairement les touristes, s'adresse également aux résidents et aura ainsi un impact sur les représentations qu'ils ont de leur ville.

\section{Cadres conceptuels}

\section{Patrimonialisation et mise en tourisme}

Si Olivier Lazzarotti aborde le tourisme et le patrimoine comme deux phénomènes co-constitutés (2011), ils répondent en effet à une même 
logique, principalement à deux niveaux. Tout d'abord, alors que Leniaud souligne que « le patrimoine n'existe pas a priori »(Leniaud, 1992, p. 3), qu'il est un construit et non un donné, nous considérons la même logique applicable au tourisme (Lazzarotti, 2003). En ce sens nous préférons aborder les processus que sont la mise en tourisme et la patrimonialisation (ou mise en patrimoine). Ainsi, s'attarder à la «mise en tourisme » plutôt qu'au tourisme permet d'éviter un certain déterminisme qui a longtemps marqué les travaux s'intéressant à cet objet de recherche. Si un lieu devient touristique, c'est bien qu'une partie de la population locale souhaite ce type de développement. Là encore, la même logique peut s'appliquer à la mise en patrimoine. Même si la patrimonialisation d'un monument, d'un site ou d'un secteur de ville est validée à un niveau national ou supranational, elle ne peut se faire sans l'aval des pouvoirs locaux. En revanche, il serait naïf de croire que ces pouvoirs locaux sont représentatifs de l'ensemble de la population locale et que des luttes, des conflits, ou simplement des points de vue divergents sont inexistants, et ce tant au niveau de la mise en tourisme que de la patrimonialisation.

\section{Touristes et résidents : des rapports à l'espace-temps différenciés}

Ayant choisi de traiter des représentations des touristes et des résidents, il nous paraît ici nécessaire de faire un court retour sur des éléments de définition. L'objectif n'est pas de revenir sur les grands débats qui ont opposés (et opposent encore) le voyageur digne de ce nom au touriste, cet « idiot du voyage »(Urbain, 1993), ni de rendre compte de façon exhaustive des définitions du tourisme et du touriste, mais simplement de s'attarder sur ce qui nous permet de distinguer le touriste d'autres individus. Un élément de définition, primordial dans le cadre de ce travail, est que le touriste se situe dans un espace-temps qui est celui du hors-quotidien (Équipe MIT, 2002), ce qui le distingue des résidents des lieux qu'il visite, qui sont eux, dans un espace-temps du quotidien. Cet élément nous paraît central, puisqu'il constitue l'une des seules différences de base entre les deux groupes. Aussi nous préférons ici le terme « résident » à celui d'« habitant » puisque nous considérons que les individus des deux groupes habitent la ville, selon des temporalités différentes.

\section{La notion de représentation}

Les représentations qui nous intéressent ici peuvent être définies comme les images résultant d'un processus de décodage par un individu d'une réalité concrète (Michelin, 1998). Il est ainsi communément admis que les représentations relèvent de chaque individu et de son contexte social. Elles sont nécessairement non-objectives et sont intimement liées aux aspects affectifs et émotionnels (Bailly et al., 1995). En ce sens, « des gens appartenant à des groupes différents peuvent se faire une image largement différente de la même réalité extérieure » (Lynch, 1998, p. 154). On pourrait ainsi croire que les représentations des touristes et des résidents seront forcément différentes. On peut alors se demander si l'image de destination peut être assez puissante pour annihiler cette subjectivité. Peut-elle engendrer une certaine uniformisation des représentations des individus relevant de ces deux groupes?

\section{La photographie comme outil d'enquête}

La volonté de donner autant d'importance aux résidents qu'aux touristes a nécessité de réfléchir à une démarche qui puisse être applicable à ces deux groupes. Hatt et al. (2011) ont démontré combien la mise en œuvre de techniques d'enquêtes classiques en sciences sociales, comme le questionnaire ou l'entretien, auprès de touristes, pouvait s'avérer difficile et peu efficace. Il semble que le temps des vacances, temps utilisé pour se « recréer » (Équipe MIT, 2002), soit peu propice à toute activité qui s'affiche comme sérieuse (enquête, recherche, etc.). La photographie, intimement liée au tourisme (Urry, 1990 ; Robinson, Picard, 2009) par son aspect ludique, nous a alors semblé constituer un outil d'enquête intéressant auprès des touristes, mais également des résidents.

La suite de cet article repose sur les résultats d'un travail de terrain effectué au printemps 2013, lors duquel nous avons rencontrés 12 couples ou familles en séjour touristique à Dinan, ainsi que 14 résidents de Dinan (tableaux 1 et 2). Pour les 


\begin{tabular}{|c|c|c|c|c|}
\hline $\begin{array}{l}\text { Code utilisé } \\
\text { dans le texte }\end{array}$ & Genre / âge & Mode (lieu) de rencontre & $\begin{array}{l}\text { Lieu de résidence habi- } \\
\text { tuelle (tel que mentionné } \\
\text { par les individus enquêtés) }\end{array}$ & $\begin{array}{c}\text { Type d'hébergement lors } \\
\text { du séjour }\end{array}$ \\
\hline T01 & Couple / $60+$ & Visite guidée & Paris & Gîte \\
\hline T02 & Couple / 50-59 & Visite guidée & Le Mans & $\begin{array}{c}\text { Appartement prêté par un } \\
\text { ami }\end{array}$ \\
\hline T03 & $\begin{array}{c}\text { Femme / 40-49 (avec } \\
3 \text { enfants) }\end{array}$ & Visite guidée & Finistère & Échange de maison \\
\hline T04 & Couple / 50-59 & Dans la rue & Montréal & Hébergés chez un ami \\
\hline T05 & Couple / 50-59 & Auberge de jeunesse (AJ) & Bordeaux & AJ \\
\hline T06 & Couple / 40-49 & AJ & Paris & AJ \\
\hline T07 & $\begin{array}{l}\text { Couple / 40-49 (avec } \\
2 \text { enfants) }\end{array}$ & Visite guidée & Angers & $\begin{array}{c}\text { Appartement prêté par un } \\
\text { ami }\end{array}$ \\
\hline T08 & Couple / 50-59 & Visite guidée & Brive-la-Gaillarde & Hôtel \\
\hline T09 & Couple / 30-39 & Nuit des musées (château) & Lille & Gîte \\
\hline T10 & Couple / 40-49 & Nuit des musées (château) & Paris & Hébergés chez un ami \\
\hline T11 & Couple / 30-39 & Nuit des musées (château) & Le Puy-en-Velay & Hôtel \\
\hline T12 & Couple / $60+$ & Dans la rue & La Roche-sur-Yon & Hôtel \\
\hline
\end{tabular}

Tableau 1 : Caractéristiques relatives aux touristes rencontrés Features related to tourists encountered

\begin{tabular}{|c|c|c|c|c|c|}
\hline $\begin{array}{l}\text { Code utilisé } \\
\text { dans le texte }\end{array}$ & $\begin{array}{l}\text { Genre /âge / statut } \\
\text { professionnel }\end{array}$ & $\begin{array}{c}\text { Natif de.../ / dernière } \\
\text { ville habitée avant } \\
\text { Dinan (si applicable) }\end{array}$ & $\begin{array}{l}\text { Année d'installa- } \\
\text { tion à Dinan }\end{array}$ & $\begin{array}{l}\text { Secteur de } \\
\text { résidence }\end{array}$ & $\begin{array}{c}\text { Statut } \\
\text { occupation }\end{array}$ \\
\hline R01 & Couple / $60+/$ retraités & Dinan & $\begin{array}{l}\text { Ne s'applique pas } \\
\left(\mathrm{N} / \mathrm{A}^{\mathrm{a}}\right) \text { (toujours } \\
\text { vécus à Dinan) }\end{array}$ & $\begin{array}{l}\text { Commune } \\
\text { voisine }\end{array}$ & Propriétaire \\
\hline R02 & F / 40-49 / commerçante & Rennes / Vienne & 1998 & $\begin{array}{l}\text { Secteur Sauve- } \\
\text { gardé }\end{array}$ & Propriétaire \\
\hline R03 & F/18-29 / employée & Dinan & N/A & Intra-muros & Locataire \\
\hline R04 & F / 60+ / commerçante & Dinan & N/A & $\begin{array}{l}\text { Secteur Sauve- } \\
\text { gardé }\end{array}$ & Propriétaire \\
\hline R05 & $\mathrm{H} / 60+$ / commerçant & Dinan & N/A & Intra-muros & Propriétaire \\
\hline R06 & $\begin{array}{c}\mathrm{H} / 50-59 / \text { travailleur } \\
\text { autonome }\end{array}$ & Normandie / Toulouse & 1997 & $\begin{array}{l}\text { Commune } \\
\text { voisine }^{\mathrm{b}}\end{array}$ & Propriétaire \\
\hline R07 & F / 30-39 / employée & Dinan & N/A & Ville & Propriétaire \\
\hline R08 & H / 30-39 / commerçant & Paris & 2008 & Intra-muros & Propriétaire \\
\hline R09 & H / 18-29 / employé & Dinan & N/A & Ville & Locataire \\
\hline $\mathrm{R} 10$ & F / 30-39 / employée & Jersey / Le Mans & 2008 & Ville & Locataire \\
\hline R11 & $\begin{array}{c}\text { F / 40-49 / travailleur } \\
\text { autonome }\end{array}$ & Rennes / Londres & 1997 & $\begin{array}{l}\text { Commune } \\
\text { voisine }\end{array}$ & Propriétaire \\
\hline $\mathrm{R} 12$ & H / 40-49 / employé & Brest & 1990 & $\begin{array}{l}\text { Commune } \\
\text { voisine }\end{array}$ & Locataire \\
\hline $\mathrm{R} 13$ & H / 60+ / retraité & Dinan & N/A & Intra-muros & Locataire \\
\hline R14 & F / 30-39 / commerçante & Istanbul & 2011 & $\begin{array}{l}\text { Commune } \\
\text { voisine }\end{array}$ & Locataire \\
\hline
\end{tabular}

Tableau 2 : Caractéristiques des résidents rencontrés

Features related to inhabitants encountered

${ }^{a}$ La mention N/A (non applicable) est utilisée pour les enquêtés natifs de Dinan, et ce afin d'alléger le tableau.

${ }^{\mathrm{b}}$ Les « communes voisines» font partie de la Communauté de communes de Dinan (CODI). 


\begin{tabular}{|l|l|l|}
\hline & \multicolumn{1}{|c|}{ Touristes } & \multicolumn{1}{|c|}{ Résidents } \\
\hline Question 1 & $\begin{array}{l}\text { Si vous deviez sélectionner une seule photographie de ce qui représente le mieux Dinan, pour la montrer à } \\
\text { quelqu'un qui ne connaît pas la ville par exemple, laquelle choisiriez-vous? }\end{array}$ \\
\hline Question 2 & Quelle photographie représente le mieux, selon vous, le patrimoine de Dinan? \\
\hline Question 3 & Quelle photographie représente le mieux, selon vous, le tourisme à Dinan? \\
\hline Question 4 & $\begin{array}{l}\text { Si, parmi les photographies de la ville que } \\
\text { vous avez prises, vous ne pouviez en conser- } \\
\text { ver qu'une seule, en souvenir de Dinan, } \\
\text { laquelle choisiriez-vous? }\end{array}$ & $\begin{array}{l}\text { Si vous deviez partir définitivement de Dinan, et que vous ne } \\
\text { pouviez emportez avec vous qu'une seule photographie de la } \\
\text { ville, que prendriez-vous en photo? }\end{array}$ \\
\hline
\end{tabular}

Tableau 3 : Questions «photographiques » adressées aux résidents et touristes

"Photographic questions" aimed at inhabitants and tourists

deux groupes, un « questionnaire photographique » était mis en place ${ }^{4}$ (tableau 3), suivi d'un entretien semi-directif. Nous considérons en effet que la photographie ne peut être utilisée seule, qu'elle doit nécessairement être accompagnée du discours verbal du participant, puisque « [ce] qui compte, ce n'est pas la photographie elle-même, mais ce qu'elle représente pour son auteur et ce pour quoi il l'a réalisée » (Bigando, 2013).

Alors que nos trois premières questions " photographiques » avaient principalement pour but de voir si les représentations des résidents étaient similaires à celles des touristes, et si elles concordaient avec les images promues, la question quatre, très largement inspirée de la méthodologie mise en place par Eva Bigando (2006, 2008), elle-même inspirée par les travaux d'Yves Michelin (1998), portant sur les paysages ordinaires, a été choisie pour dégager des pistes quant à l'impact de « l'image touristique patrimoniale » sur les représentations qu'ont les résidents. Ce thème, identifié par Bigando comme «paysage intime », a dans le cadre de ses travaux permis de produire les photographies les plus personnelles, avec une très forte représentation de la sphère privée (maison, jardin, vue depuis chez-soi, etc.) Cette question visait en partie à apporter des éléments de réponse à l'une de nos interrogations de départ, à savoir si les lieux du quotidien, considérés comme banals, étaient élevés au rang de lieux extra-ordinaires par les mises en tourisme et en patrimoine.

4. Seules les quatre premières questions seront abordées dans cet article.

\section{UNE PETITE VILLE HISTORIQUE MISE EN TOURISME ET EN PATRIMOINE}

Plusieurs travaux de recherche portant sur le «tourisme de patrimoine » ont mis en avant les « ravages » du tourisme sur la vie locale, les touristes venant nécessairement perturber le milieu de vie des résidents. Toutefois, ceux-ci prennent le plus souvent comme exemples des lieux touristiques emblématiques, pouvant être qualifiés de « hautslieux », comme Venise (Russo, 1999), ou encore la Cappadoce (Tucker, Emge, 2010). Mais cette opposition peut-elle être généralisée à l'ensemble des lieux « historiques » recevant des touristes? Il convient donc d'expliciter brièvement en quoi la ville que nous désignons comme " petite ville historique » se distingue de ces autres lieux mis en tourisme et en patrimoine.

D'abord, comme l'écrivait Maria Gravari-Barbas à propos de la ville moyenne : «par son échelle, [elle] permet au visiteur d'appréhender son héritage historique et architectural de manière plus globale, de participer à une expérience totale » (Gravari-Barbas, 1997). Ainsi à la différence de certaines grandes villes, telles Québec ou Prague, souvent prises en exemple en termes de tourisme patrimonial, où le patrimoine qui attire les visiteurs s'inscrit dans un seul ou quelques quartiers bien distincts de la ville ${ }^{5}$, ce même patrimoine englobe, à l'échelle de la petite et moyenne ville, l'ensemble de la ville centre. En second lieu, contrairement à certaines petites communes portées au rang de « hauts-lieux » du tourisme,

5. Le Vieux-Québec pour la première, Hradčany et Staré Město pour la seconde. 
en grande partie par l'acquisition du label international Patrimoine mondial de l'humanité inscrit par $l^{\prime} U_{n e s c o}{ }^{6}$, la ville qui nous intéresse ici, bien que disposant d'un patrimoine architectural important, ne bénéficie que d'une reconnaissance de son patrimoine aux niveaux régional et national. Cette reconnaissance d'une portée plus réduite explique, en partie, qu'elle capte des flux touristiques beaucoup moins importants. Les travaux d'Olivier Lazzarotti (2000, 2011) ont su démontrer l'existence de liens forts entre une labellisation patrimoniale à l'Unesco et l'importance des flux touristiques.

Alors que dans ces cas emblématiques, nous sommes face, que ce soit à l'échelle d'un quartier dans le premier cas de figure ou de l'ensemble de la commune dans le second, à ce que certains auteurs ont appelé une monoculture touristique (Cazes, 1998; Gravari-Barbas, 2001), la « petite ville historique » qui nous intéresse ici se démarque par le maintien d'une mixité des fonctions.

\section{L'exemple dinannais}

«Une belle vieille ville, agglutinée [...] sur un précipice, comme un nid d'hirondelle ${ }^{7}$. »

Au cœur d'une région qui attire principalement les touristes pour ses balades (à pied ou à vélo), ses plages ou encore la visite de sites naturels ${ }^{8}$, les villes historiques bretonnes, essentiellement petites ou moyennes ${ }^{9}$, se sont regroupés en association (Union des Villes d'Art et d'Histoire et des Villes Historiques de Bretagne), afin de promouvoir leur patrimoine bâti. Dinan compte parmi les pionnières, étant présente dès la création de l'association en 1984, son maire en assurant par ailleurs la présidence ${ }^{10}$.

Cette petite ville bretonne d'environ 11000 habitants collectionne en effet les éloges sur son patrimoine. La ville, qui jouit d'un positionnement privilégié, à proximité de « hauts-lieux » du tourisme (Mont-Saint-Michel à une soixantaine de kilomètres et Saint-Malo à environ 35 km), conserve la

6. On peut citer ici Göreme (Cappadoce) ou encore le Mont-Saint-Michel.

7. Victor Hugo (1836), En voyage: France et Belgique.

8. Comité Régional du Tourisme (CRT) - Bretagne, document « Le tourisme en Bretagne », p. 29, http://acteurs.tourismebretagne.com/vous-informe/ chiffres-du-tourisme-observatoire, consulté le 4 juin 2014.

9. Des villes membres, seules Rennes et Nantes comptent plus de 100000 habitants.

10. Entretien avec monsieur René Benoît (mars 2013), alors maire de Dinan et président de ladite association. quasi-totalité de son rempart, tours et portes ( $\mathrm{XIII}^{\mathrm{e}}$ $\mathrm{XVII}^{\mathrm{e}}$ siècle). Cette situation lui permet d'attirer des flux touristiques importants, soit entre 130000 et 140000 visiteurs annuellement ${ }^{11}$.

À une trentaine de kilomètres de la côte, son patrimoine urbain constitue son principal «produit d'appel »(figure 1). Celui-ci bénéficie d'une reconnaissance à l'échelle nationale, puisque la ville possède un Secteur Sauvegardé depuis 1996 et est Ville d'Art et d'Histoire (VAH) depuis 1984. Malgré la diversité du patrimoine bâti dinannais comprenant, entre autres, des maisons en-pan-de-bois, des hôtels particuliers, de nombreux couvents, couvrant une période allant des $\mathrm{XII}^{\mathrm{e}}$ au $\mathrm{XX}^{\mathrm{e}}$ siècles, l'" image de marque »qui lui est le plus souvent associée demeure celle de "cité médiévale ». Même si on affirme, tant au service du Patrimoine de la Ville qu'au bureau du développement touristique de la communauté de communes (CODI), vouloir se défaire de cette image de cité médiévale, l'attitude des responsables est quelque peu ambivalente: "Vous prenez nos maisons à pans de bois, qui constituent un élément vraiment marquant du patrimoine de la ville, sur ces 110 maisons, vous en avez 10 de médiévales. [...] Vous prenez les couvents, ils sont du XVII siècle, etc., etc. À part le rempart finalement, Dinan "cité médiévale", c'est une image d'Épinal. Nous, on essaie d'en sortir ${ }^{12}$."

Comme le souligne ces propos du responsable du service du Patrimoine, on souhaite se détacher de cette image, mais sachant que c'est en grande partie celle-ci qui attire les touristes, on continue de la promouvoir, comme en témoigne le thème des visites contées mises en place cette année-là par ce même service : «Contes et fabliaux du Moyen Âge ».

De même, alors que plusieurs auteurs ont bien démontré qu'inévitablement, la mise en patrimoine "implique des tris, des choix donc des oublis » (Lazzarotti, in Lévy et Lussault, 2003, p. 693), Dinan n'y fait pas exception. À la mairie, on nous affirme ne rien vouloir détruire. Pourtant, la ville a, à plusieurs reprises au cours des dernières années, fait usage de son droit de préemption pour l'achat et la démolition de maisons jouxtant les remparts.

11. Ces données chiffrées correspondent au nombre de passages à l'Office de Tourisme. Elles sont uniquement fournies à titre indicatif, et ce afin de brosser un portrait très général et non exhaustif de l'activité touristique à Dinan.

12. Propos du responsable du Service Patrimoine de la Ville en entretien (février 2013). 

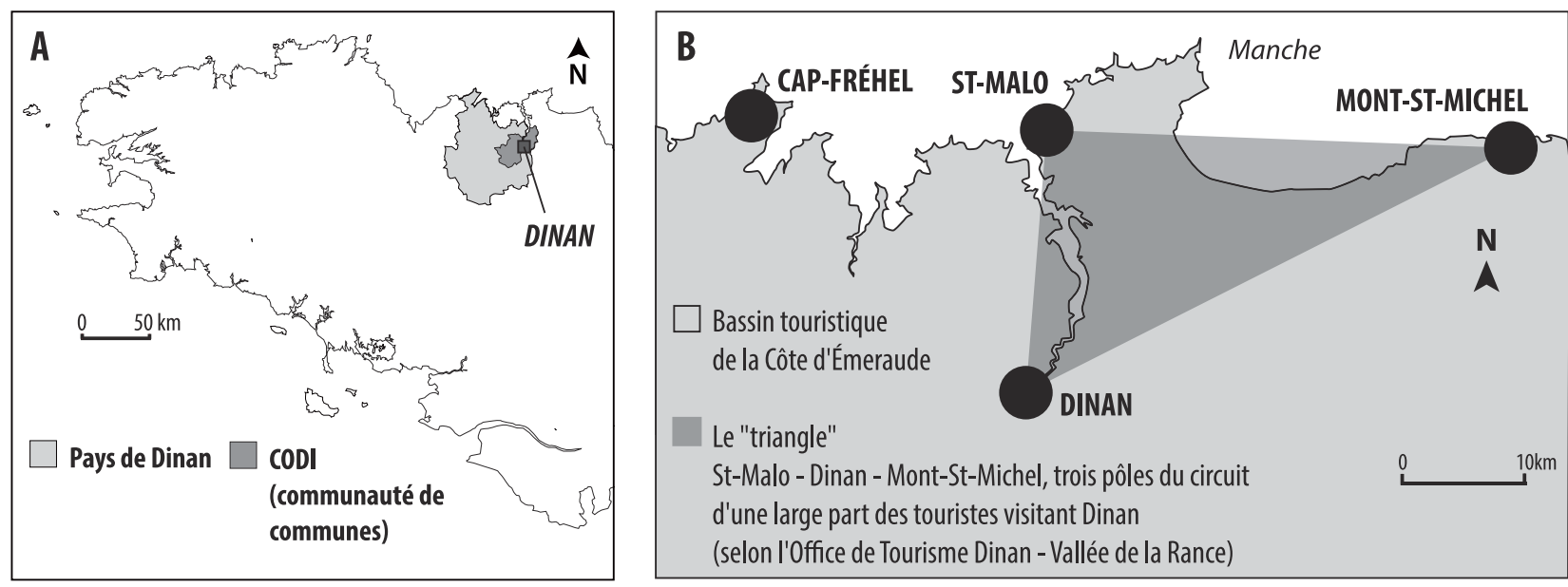

Réalisation: Annie Ouellet, 2014

Figure 1 : Localisation de Dinan et de sa communauté de communes (CODI) en Bretagne (A) et au sein du bassin touristique de la Côte d'Êmeraude (B).

Localisation of Dinan and the community of municipalities (CODI) in Brittany (A) and in the touristic zone of the "Côte d'Émeraude" (B)

On retrouve d'ailleurs la plupart de ces opérations sous le titre "Dégagement et mise en valeur des remparts ${ }^{13}$ », dans les registres de délibération des archives municipales. Parmi celles-ci on retrouve la « maison Tampe » au 9 rue Michel qui a été démolie au profit de l'ouverture du chemin de ronde (1988), la «maison du Puisatier » qui a été démolie pour ouvrir le passage de la Tour de l'Horloge (1994) et plus récemment, les $n^{\circ} 8$ et 10 de la rue du Général de Gaulle (2011), ces maisons datant toutes des XIX ${ }^{\mathrm{e}}$ et $\mathrm{Xx}^{\mathrm{e}}$ siècle. Il est toutefois important de noter que même si ces décisions relèvent de l'administration municipale, elles se sont prises, à chaque fois, avec l'accord des représentants des instances patrimoniales aux niveaux régional et national.

En valorisant essentiellement son passé médiéval, Dinan s'inscrit dans un principe de « réduction narrative » (Vlès, Berdoulay, Clarimont, 2005). Son histoire est simplifiée, mettant l'accent sur quelques passages particulièrement glorieux, qui permettent de distinguer et mettre en valeur la ville.

\section{SUIVRE LE GUIDE...}

« Le tourisme est venu s'ajouter aux autres préoccupations des hommes : comme pour la décla-

13. On retrouve cette expression pour nommer plusieurs affaires traitées, entre autres, lors des séances du Conseil du 31 juillet 1986, 30 septembre 1988, 7 février 1994 (liste non exhaustive). ration d'impôts, l'inscription des enfants à l'école, l'achat d'un appartement, il existe des guides pour le tourisme; des guides, donc une sélection du message. » (Miossec, 1977 :64)

Les guides, suivant les propos de Miossec, par une sélection de ce qui doit être «vu » et «fait» lors d'un séjour dans une ville touristique, induisent inévitablement une certaine orientation du regard du touriste. Ces guides peuvent prendre de multiples formes. Nous nous sommes ici concentrés sur trois types : les guides de voyage imprimés ${ }^{14}$, les cartes postales ${ }^{15}$ et l'Office de tourisme $(\mathrm{OT})^{16}$. L'objectif n'est pas d'établir une liste exhaustive dans le but d'en dégager des données chiffrées, mais plutôt d'avoir une vue d'ensemble. Arrivent ainsi en tête les rues du Jerzual ${ }^{17}$, de l'Horloge, de l'Apport, la place des Mercier, le château, le rempart, la basilique Saint-Sauveur, le port et la Tour de l'Horloge (figure 2).

Les photographies prises par les touristes rencontrés et sélectionnées pour répondre à nos questions

14. Six guides ont été utilisés : Géoguide, Guide Évasion, Guide Voir, Guide Gallimard, Le Petit Futé, Guide Michelin.

15. Un inventaire des cartes postales en vente chez cinq marchands du centre historique a été fait, totalisant 78 modèles de cartes représentant Dinan (nous avons exclus celles représentant des images "génériques » de la Bretagne).

16. Plan touristique offert à tous les touristes se rendant à l'OT ( $c f$. figure 2).

17. La rue portant le nom de Jerzual dans sa partie intra-muros prend le nom de rue du Petit-Fort dans sa partie extra-muros. Néanmoins, la rue est le plus souvent désignée dans son ensemble par son appellation « Jerzual ». 


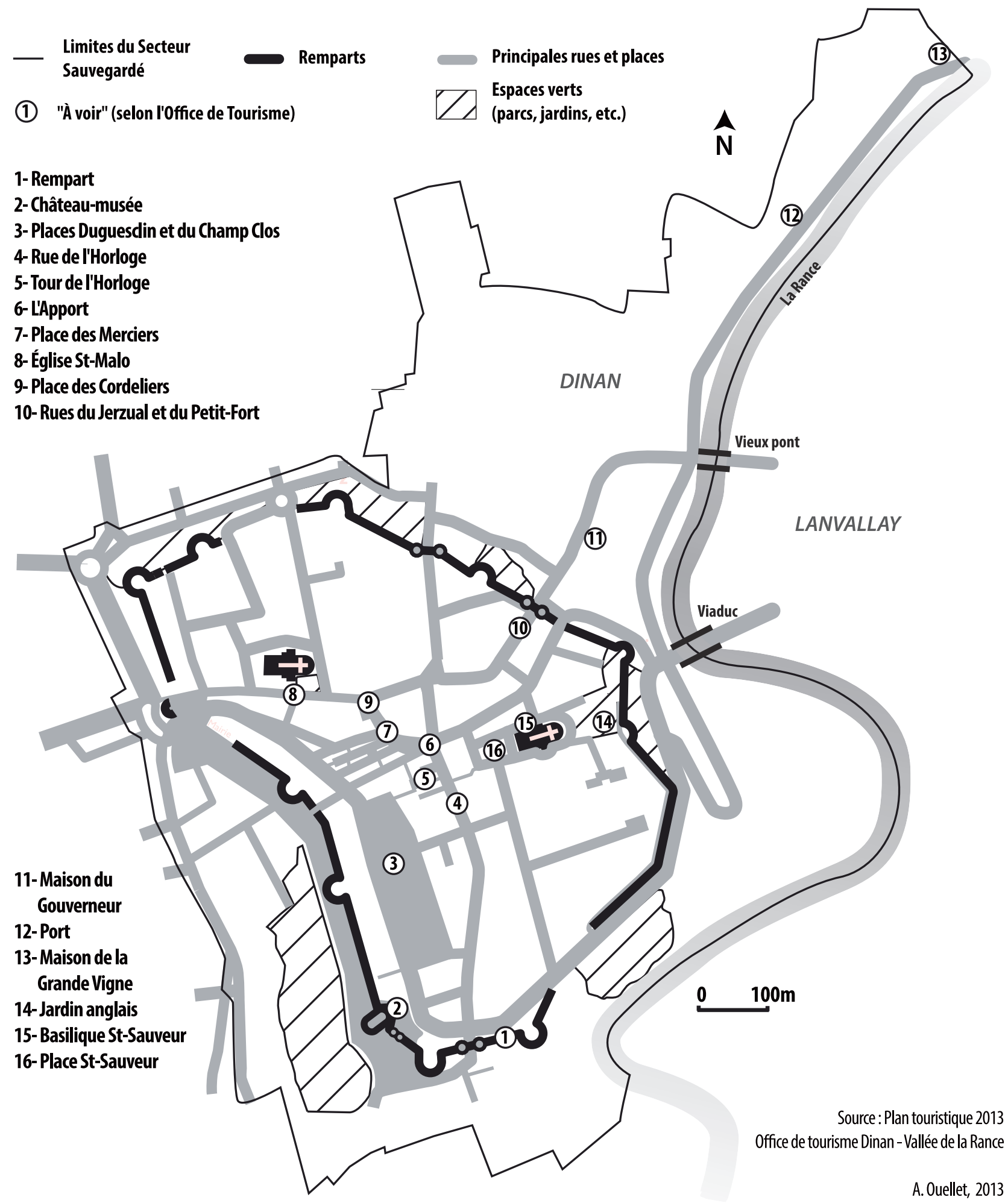

Figure 2 : Patrimoine et tourisme à Dinan. Lieux et monuments « à voir » selon l'Office de Tourisme. Héritage and tourism in Dinan. "Must see" places and monuments according to the Tourism Office. 
correspondent globalement à ces mêmes lieux ou monuments.

Plusieurs clichés nous ayant été fournis sont par ailleurs quasi identiques aux cartes postales représentant les mêmes lieux (figure 3), cette forte similitude confirmant le principe du « cercle herméneutique ${ }^{18}$ » évoqué par Chris Ryan, celui-ci affirmant que «le touriste recherche ce qui a été photographié dans le but de reproduire les mêmes images » (Ryan, 2002) ${ }^{19}$.

\section{Dinan, une ville « pittoresque et charmante ${ }^{20}$ »}

L'analyse des photographies choisies par les touristes fait apparaître de nombreux clichés jumeaux, le plus souvent, en réponse aux questions une et quatre (ce qui représente le mieux la ville et le cliché à garder en souvenir de la ville). Il semble que davantage qu'un détail architectural, un lieu particulier, c'est ce qui représente la ville dans son ensemble que les touristes souhaitent conserver en souvenir. Ceci s'inscrit en parfaite cohérence avec le discours tenu par la plupart des participants, affirmant avoir beaucoup de mal à choisir parmi leurs clichés, puisque " tout est magnifique» (T05), et que "ce n'est pas une rue ou un monument en particulier qui nous a plu, c'est la ville dans son ensemble... » (T12)
Néanmoins, certaines rues se voient surreprésentées. La rue du Jerzual compte parmi celles-là (figure 4). Elle a été choisie par plus de la moitié des touristes rencontrés et est souvent utilisée pour répondre à deux, voire trois questions. Les touristes nous disent apprécier l'aspect des maisons qui la bordent mais surtout l'ambiance générale qui y règne symbolisant l'aspect authentique et pittoresque de la ville, avec les nombreux artisans qui y ont pignon sur rue.

Le château est très présent dans l'image de la ville. Il est l'un des monuments le plus souvent évoqué dans les guides touristiques. Nous aurions ainsi pu nous attendre à une forte représentation de celuici parmi les clichés des touristes. Or, assez peu de touristes rencontrés nous en ont fourni. Seuls trois couples l'ont choisi, dont deux lors de la Nuit européenne des musées. Les ayant rencontrés suite à la visite guidée du château, le contexte pouvait entrâ̂ner un biais, comme l'a d'ailleurs fait remarquer l'un d'eux : "Ce qui représente le mieux la ville... Ce serait sûrement le châtean et les remparts... parce que des ruelles avec des maisons à colombage, ça on en trouve dans plein de villes, alors que le château, c'est plus rare! [...] mais on n'est peut-être pas très objectifs étant donné qu'on vient juste de le visiter... » (T10)
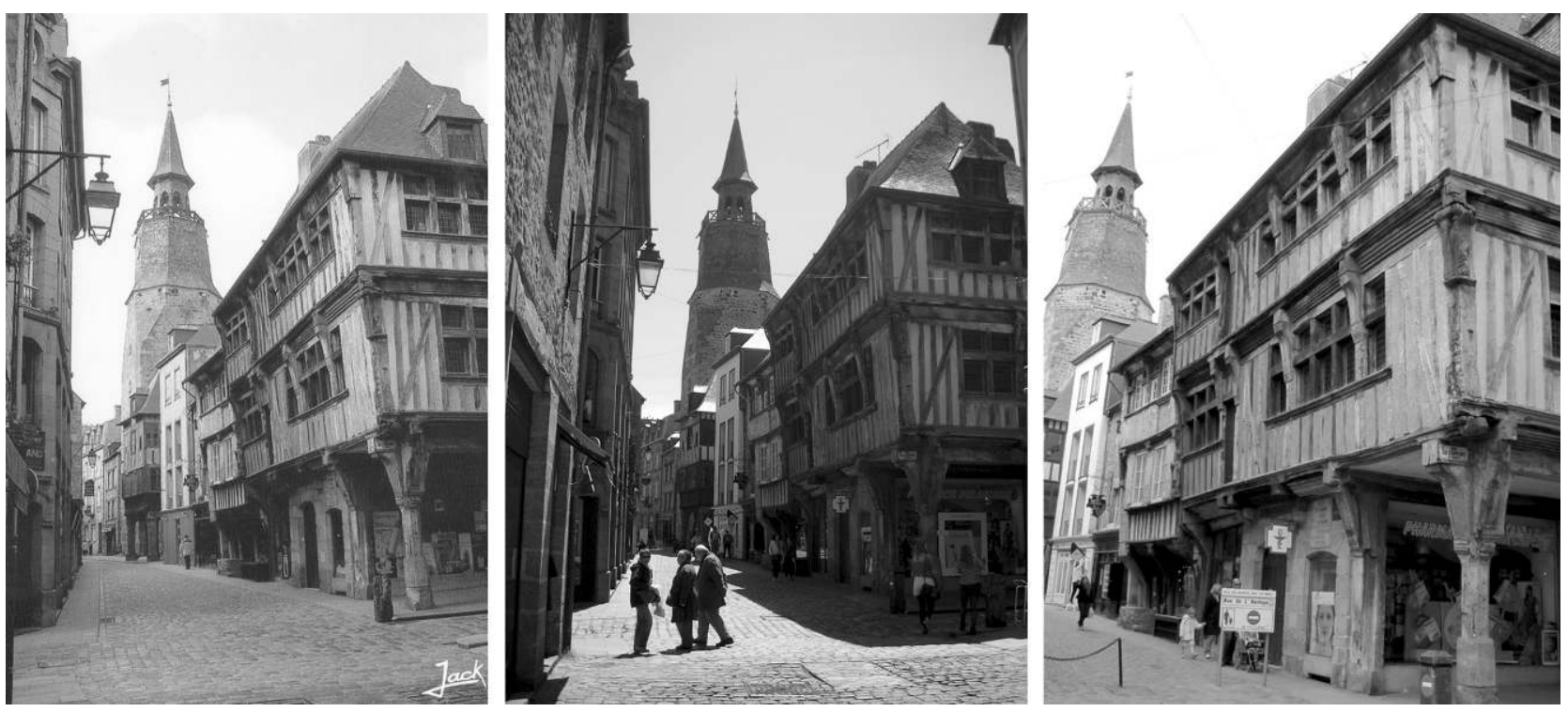

18. «Hermeneutic circle».

19. «The tourists seeks what has been photographed in order to photograph it »-traduction personnelle.

20. Propos d'un touriste rencontré à Dinan (T05).
Figure 3 : Rue de l'Horloge. Carte (éditions Jack) et photos de touristes recueillies lors de l'enquête (réponses à la question 2).

"Rue de l'Horloge". Postcards (Jack's Edition) and pictures taken by tourists (answers to question number 2). 

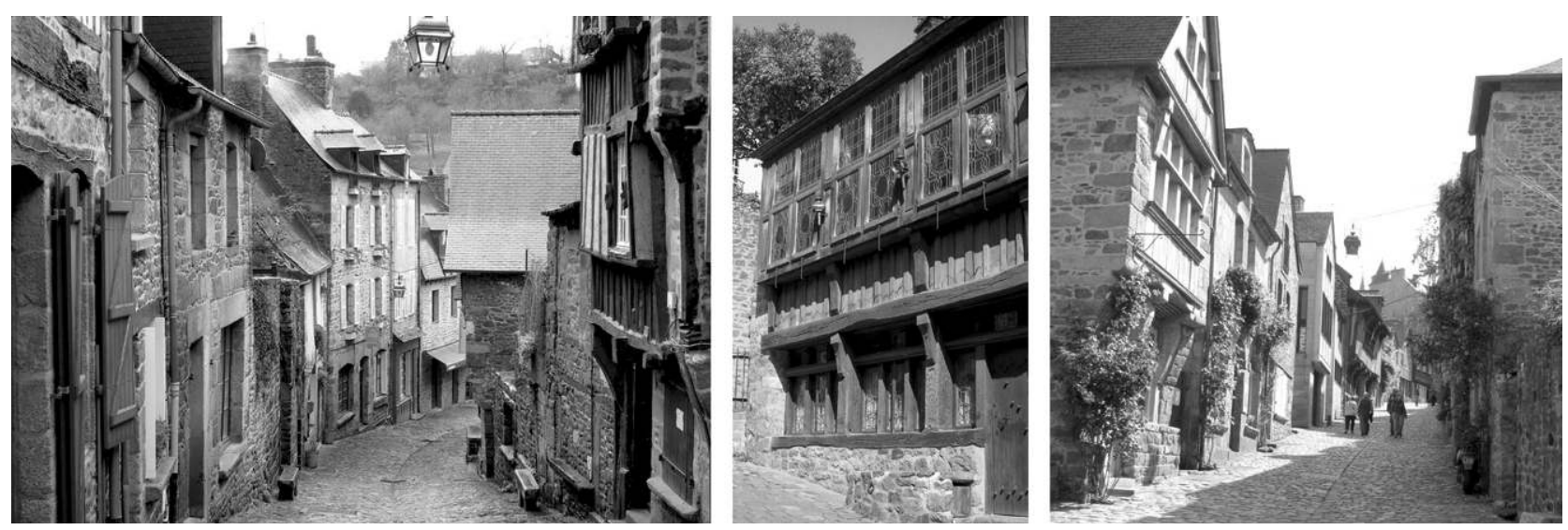

Figure 4: La rue du Jerzual. Photographies prises par des touristes "Rue du Jerzual". Pictures taken by tourists

Alors que ce touriste précise que le château et le rempart sont ce qui représente le mieux la ville, puisqu'étant plus rare que les maisons à colombages, d'autres sont d'avis contraire : "Je dirais une des photos avec toutes les maisons anciennes. C'est vraiment l'ensemble des maisons qui est typique... parce qu'un château ou des remparts, on peut en trouver ailleurs, mais c'est vraiment le fait que tout le centre ait encore ses maisons à colombages, c'est ça qui est caractéristique. » (T01)

Ces extraits témoignent bien de la diversité des représentations possibles. Malgré les balises établies par les guides, chacun conserve tout de même sa « façon de voir ». Aussi, ils tendent à conforter l'idée que la rareté génère un attrait particulier. Dans les deux cas, ce qui est le plus représentatif est ce qui est le plus rare, et c'est ce qu'ils recherchent, en tant que touristes. La représentation de la ville, dans ces cas, serait ainsi liée à son caractère distinctif.

\section{Une représentation du tourisme très lucide}

Notre question liée à la représentation du tourisme, à laquelle nous pensions obtenir des réponses photographiques très proches des images projetées, a généré, à l'inverse, des réponses qui, dans la majorité des cas, s'en éloignaient largement. Le tourisme est le plus souvent représenté par des commerces divers, que ce soit des boutiques, artisans, restaurants. Appréciant les "petites rues piétonnes avec plein de boutiques et d'artisans » (T10), ils réalisent être dans un lieu qui leur est prioritairement dédié, comme le souligne cette Finistérienne : «La rue du Jerzual avec tous les artisans, les boutiques, c'est certainement ce qui représente le mieux le tourisme, parce que j'imagine bien qu'il n'y en aurait pas autant si c'était pas touristique. Il doit y avoir assez peu de gens d'ici qui achètent dans ces boutiques. » (T03)

Chez ces touristes, la forte présence de ces commerces n'est pas perçue négativement. En étant touristes, c'est ce type d'ambiance et de lieux qu'ils recherchent, tout en ayant conscience (à divers degrés) qu'ils ne sont sûrement pas ceux que les « locaux» fréquentent. Quelques participants ont quant à eux opté pour des lieux particulièrement attractifs pour les touristes, comme la rue du Jerzual et l'Apport.

Alors que le fait de demander à voir les photographies des touristes nous paraissait un moyen efficace d'entrer en interaction avec eux, notre expérience nous a montré que la photographie, chez plusieurs touristes rencontrés, suscitait beaucoup moins d'intérêt que ce à quoi nous nous étions attendus. Ils étaient fréquemment beaucoup plus enclins à nous parler de leurs impressions et ressentis par rapport à la ville, qu’à montrer leurs clichés.

\section{Le discours verbal pour dépasser le « cercle herméneutique "}

Le discours verbal des touristes nous permet ainsi de pousser plus loin l'analyse que leur seul « discours photographique ». Plusieurs touristes nous ont d'ailleurs précisé avoir eu du mal à choisir ce qui représentait le mieux chacun des éléments qui leurs étaient demandés. Pour eux, leur sentiment général par rapport à la ville semblait être plus impor- 
tant. Plus que des lieux précis, les questions liées à l'ambiance de la ville, à son caractère authentique sont fréquemment apparues au cours de l'entretien. Quelques-uns ont abordé le sujet de la ville-musée, le plus souvent en comparant Dinan à d'autres villes. Trois couples ont évoqué Saint-Malo et le MontSaint-Michel, visités au cours des jours précédant leur passage à Dinan. Cette dernière gagne généralement au jeu de la comparaison :

"Saint-Malo c'est joli, on ne peut pas dire le contraire... mais c'est froid... il n'y a pas d'âme. Ici on sent qu'il y a une âme, que c'est vivant. » (T01)

"Le centre historique est sympa, mais surtout ce qui est bien c'est qu'on sent que oui il y a du tourisme, mais il n'y a pas que ça. [...] Contrairement au Mont-Saint-Michel où on était hier où là vraiment c'est que ça... il n'y a que des magasins de souvenirs partout... vraiment ça manque d'authenticité je dirais... tandis qu'ici on sent que c'est plus vrai. Ici on n'est pas dans une ville-musée, malgré le tourisme, ça reste une ville, une vraie ville. » (T08)

Ce type de discours reflète globalement celui d'une majorité de touristes enquêtés. Bien que n'ayant pas tous visité Saint-Malo ou le Mont-SaintMichel, ou ne l'évoquant tout simplement pas, il semble que Dinan soit considérée comme étant plus authentique, plus vraie, que ses voisines, pourtant beaucoup plus populaires au niveau touristique.

Toutefois, certains touristes souhaitent et recherchent cette ville-musée, comme ce touriste qui nous parle de Dinan et Locronan avec enthousiasme: "Toute la ville ancienne est magnifique. On se plonge vraiment dans une autre époque... c'est un peu comme Locronan. Vous connaissez? C'est très ancien, très typique, les gens sont habillés comme à l'époque et tout, vraiment c'est génial, c'est vraiment une ville-musée! » (T09)

Il semble que certains touristes se confortent dans la contemplation de la ville-décor, ils ne souhaitent pas en avoir l'arrière-scène ou les coulisses, le front (MacCannell, 1976) leur convient, puisque c'est ce pour quoi ils se sont déplacés. Dans ce cas, la ville semble réellement choisie non pas en tant que ville mais en tant que destination.

Ainsi, uniquement en termes de réponses photographiques, les résultats obtenus auprès des touristes tendent ainsi à invalider notre hypothèse de départ, voulant que les clichés choisis se démarqueraient de l'image de destination, puisqu'étant dans une petite ville où le tourisme ne constitue pas une monoculture. Toutefois, c'est davantage dans le discours verbal des participants que l'on retrouve cet aspect de « vraie ville » vivante et animée, ce qui nous conforte quant à notre choix de croiser les outils d'enquête que sont l'entretien et la photographie.

\section{SE REPRÉSENTER «SA 》VILLE}

« L'environnement familier, c'est ce que l'on a toujours vu sans jamais l'avoir regardé. » (Bourdieu et al., 1965)

Si on ne porte généralement pas attention à son environnement quotidien, on peut se demander, comme indiqué en introduction, si le fait que ce lieu de vie soit élevé au rang de lieu de grande qualité par les mises en tourisme et en patrimoine, modifie le regard qu'on y porte. Est-ce que les Dinannais, en tant que résidents d'une "petite ville touristique patrimoniale », portent un regard différent sur leur milieu de vie? C'est à ces questions que nous avons tenté d'apporter des éléments de réponse.

\section{Dinan et patrimoine : des synonymes?}

Pour plusieurs résidents, ce qui représente la ville et ce qui représente le patrimoine correspond à deux façons de poser la même question. La représentation de Dinan comme ville se confond avec son patrimoine. La place des Merciers et la rue de l'Apport, regroupant des maisons à pans de bois, dont certaines initialement construites au Moyen Âge, sont certainement les lieux les plus photographiés, et ce, autant pour représenter la ville que le patrimoine.

Pour une autre résidente, cette forme d'assimilation des concepts va encore plus loin : ce qui représente le mieux la ville serait aussi son patrimoine, mais un patrimoine monumental nécessairement mis en tourisme: "C'est un peu la même chose, ce qui représente la ville et ce qui représente le patrimoine. Parce que oui, le patrimoine c'est ce que la ville possède comme monuments importants. [...] pour moi t'en as deux, soit la Tour de l'Horloge soit le château, c'est les deux que les touristes peuvent visiter. » (R07)

Pour cette Dinannaise, la ville ne prendrait ainsi sens que par son caractère touristique. Cette repré- 
sentation du patrimoine sous la forme d'un monument important est aussi très présente chez d'autres résidents pour qui les représentations de la ville et du patrimoine sont bien distinctes. Pour ceux-ci, la ville est souvent représentée par une vue d'ensemble, alors que le patrimoine est un monument, classé : «Pour moi le patrimoine c'est quelque chose qui est classé, qui est important, qui est protégé... donc je dirais soit le château, ou la Tour de l'Horloge. » (R10)

Deux représentations du patrimoine tendent ainsi à se dégager. Un patrimoine vernaculaire largement assimilé à la ville dans son ensemble : le patrimoine de Dinan ce sont ses maisons à pans de bois et Dinan c'est son patrimoine bâti. La deuxième représentation du patrimoine qui émerge du discours (verbal et photographique) des Dinannais est un patrimoine monumental, reconnu comme tel et classé à l'inventaire des Monuments Historiques : la Tour de l'Horloge, la maison du Gouverneur, le château ou encore le rempart (figure 5).

\section{Ce qui représente le tourisme ou " là où les Dinannais ne vont pas »}

Si photographier ce qui représente le mieux la ville et le patrimoine semblait relativement simple pour les enquêtés, la représentation du tourisme a posé davantage de problèmes. Si une majorité a opté pour «ce que tous les touristes prennent en photo ", il est surtout apparu que ce qui représente le tourisme correspond à ce qui n'est pas approprié par les résidents, des lieux qui sont exclusivement pratiqués par les touristes. La rue du Jerzual et le château comptent parmi les réponses les plus souvent évoquées.

"Le château?! Pour le tourisme tu veux dire? Parce que moi c'est pas du tout le château que je voudrais garder comme image de la ville! Le château, on l'a visité une fois, en 40 ans! Mais oui c'est vrai que nous aussi quand on visite une ville et qu'il y a un château, on aime bien le visiter. " (R01, répondant à son mari affirmant vouloir photographier le château)

«Pour moi le tourisme, je pense que ce serait le Jerzual... Nous on n'y va jamais, c'est trop touristique. » (R07)

"C'est sûr que le Jerzual c'est un classique pour les touristes! » (R08)

En effet, le Jerzual apparaît comme particulièrement emblématique d'un lieu très apprécié par les touristes ( 9 clichés pour les questions une à trois) alors qu'il est boudé par la majorité des résidents rencontrés (un seul cliché, choisi par une résidente habitant cette rue) (tableau 4).
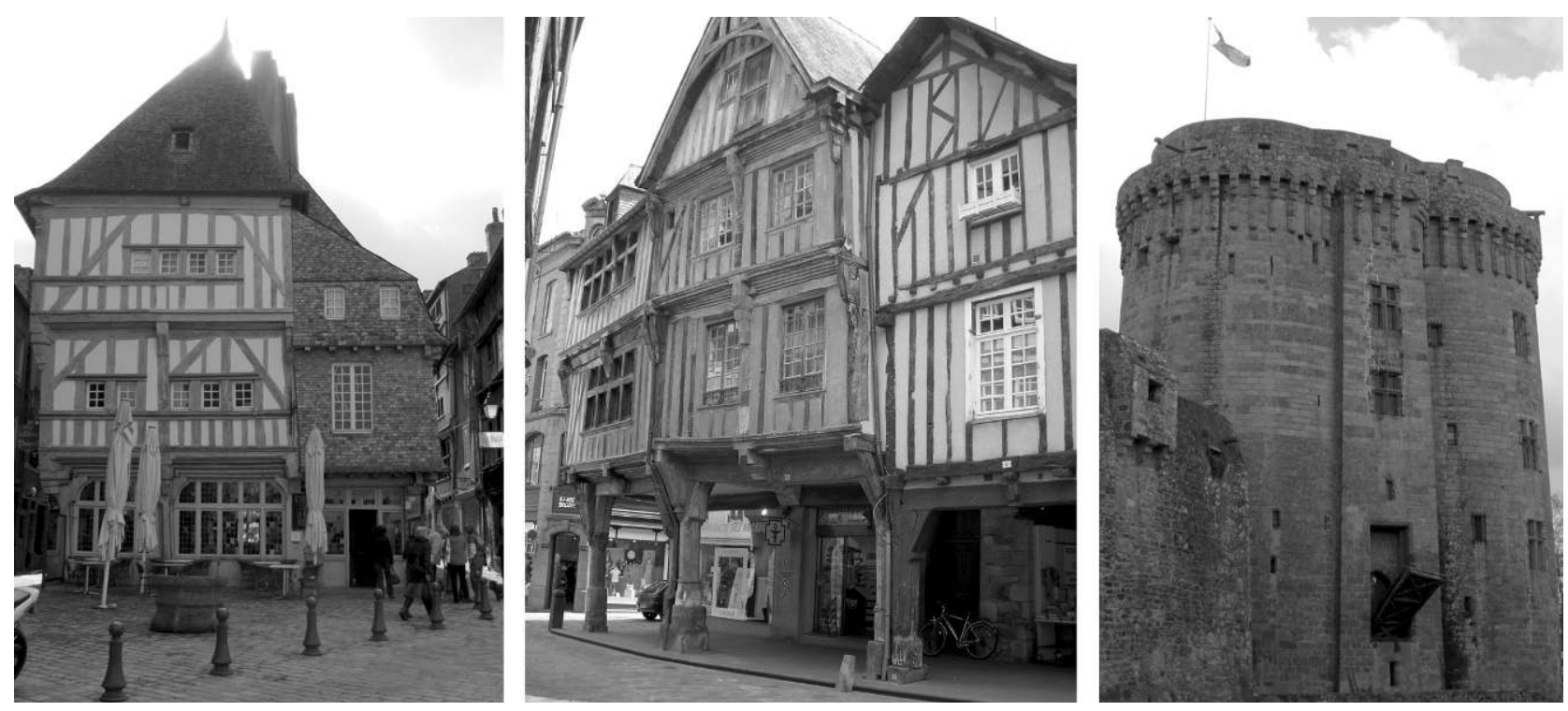

Figure 5 : Photographies de trois résidents en réponse à la question 2 (représentation de la ville) De gauche à droite : Maison de la mère Pourcel (Place des Merciers), rue de l'Apport, château. Pictures from three inhabitants as answers to question 2 (representation of the city)

From left to right: Maison de la mere Pourcel (Place des Merciers), the "rue de l'Apport ", the castle. 
Pour ces participants, résidents et touristes sont considérés comme deux catégories à part, ayant chacun des lieux qui leurs sont dédiés.

Un autre type de réponse apparaît : les commerces. Certains résidents associent ainsi le tourisme aux commerces dits «touristiques» (boutiques de souvenir, de produits locaux, etc.), là encore en opposition aux résidents, qui eux, ne les fréquentent pas. Les résidents ayant fourni cette réponse sont relativement critiques par rapport au tourisme, entre autres au fait que la ville est «envahie de magasins à touristes. »(R02). Cette question a semblé, pour ces répondants, susciter beaucoup moins d'intérêt que les autres, semblant être associée à quelque chose qui ne les concerne pas.

Bien qu'il soit quelque peu hasardeux de définir des catégories de représentations, quatre tendances peuvent être dégagées du discours des résidents par rapport au tourisme (figure 6). Il est considéré par certains comme une industrie représentée par les commerces (R03 et R12). Pour d'autres, le tourisme est associé à des lieux qui sont quasi exclusivement dédiés aux touristes (R01, R02, R06, R07, R08 et
R13). Il est presque systématiquement rappelé, par ces résidents, qu'eux ne les fréquentent pas. Une troisième tendance apparaît, associant le tourisme aux rues les plus arpentées et surtout les plus photographiées par les touristes, mais cette fois sans distinction entre touristes et Dinannais (R04, R05, R10, R14). Certains rappelant d'ailleurs que «les touristes, ils ne sont pas différents de nous, les rues que nous on trouve jolies... c'est pareil pour eux!» (R10). Finalement, pour deux résidents le tourisme symbolise la détente, le farniente (R09 et R11). Si pour ces derniers on a une association tourisme - détente, et pour les premiers, une association tourisme - commerce, pour la majorité des répondants, qu'ils identifient des lieux d'où les Dinannais sont absents ou non (deuxième et troisième tendances), le tourisme demeure largement associé au patrimoine urbain.

\section{La ville au quotidien : de l'ordinaire à l'extra-ordinaire}

Alors que les représentations de la ville, du patrimoine et du tourisme étaient globalement en adé-

\begin{tabular}{|c|c|c|}
\hline \multirow{2}{*}{ Lieux / monuments photographiés } & \multicolumn{2}{|c|}{ Nombre de clichés pour chaque groupe } \\
\hline & Résidents & Touristes \\
\hline $\begin{array}{c}\text { Rue de l'Apport / Place des Merciers } \\
\text { Nous ne différencions pas la rue de l'Apport de la place des Merciers, puisque } \\
\text { les deux forment un ensemble pensé comme un tout dans le discours de tous } \\
\text { les participants. }\end{array}$ & 10 & 8 \\
\hline Rempart & 6 & 2 \\
\hline Port / viaduc & 5 & 6 \\
\hline Rue de l'Horloge & 4 & 4 \\
\hline Tour de l'Horloge & 4 & 1 \\
\hline Vue générale sur la ville (photo prise depuis le viaduc (côté Lanvallay)) & 3 & 0 \\
\hline Château & 2 & 3 \\
\hline Rue de la Mittrie & 2 & 0 \\
\hline Statue de Du Guesclin (sur la place du même nom) & 1 & 1 \\
\hline Basilique Saint-Sauveur & 1 & 1 \\
\hline Maison du Gouverneur & 1 & 0 \\
\hline Rue de la Cordonnerie & 1 & 0 \\
\hline Rue de la Chaux & 1 & 0 \\
\hline Rue du Jerzual / Rue du Petit-Fort & 1 & 9 \\
\hline Hôtel Keratry & 0 & 1 \\
\hline Nombre total de clichés & 42 & 36 \\
\hline
\end{tabular}

Tableau 4 : Lieux et monuments photographiés par les résidents et les touristes en réponse aux questions 1 (représentation de la ville), 2 (représentation du patrimoine) et 4 (cliché à conserver en souvenir) (Source : réalisation personnelle)

Places and monuments photographed by inhabitants and tourists as answers to question 1 (representation of the city), 2 (representation of heritage) and 4 (picture to keep as a souvenir) (Source: personal research) 


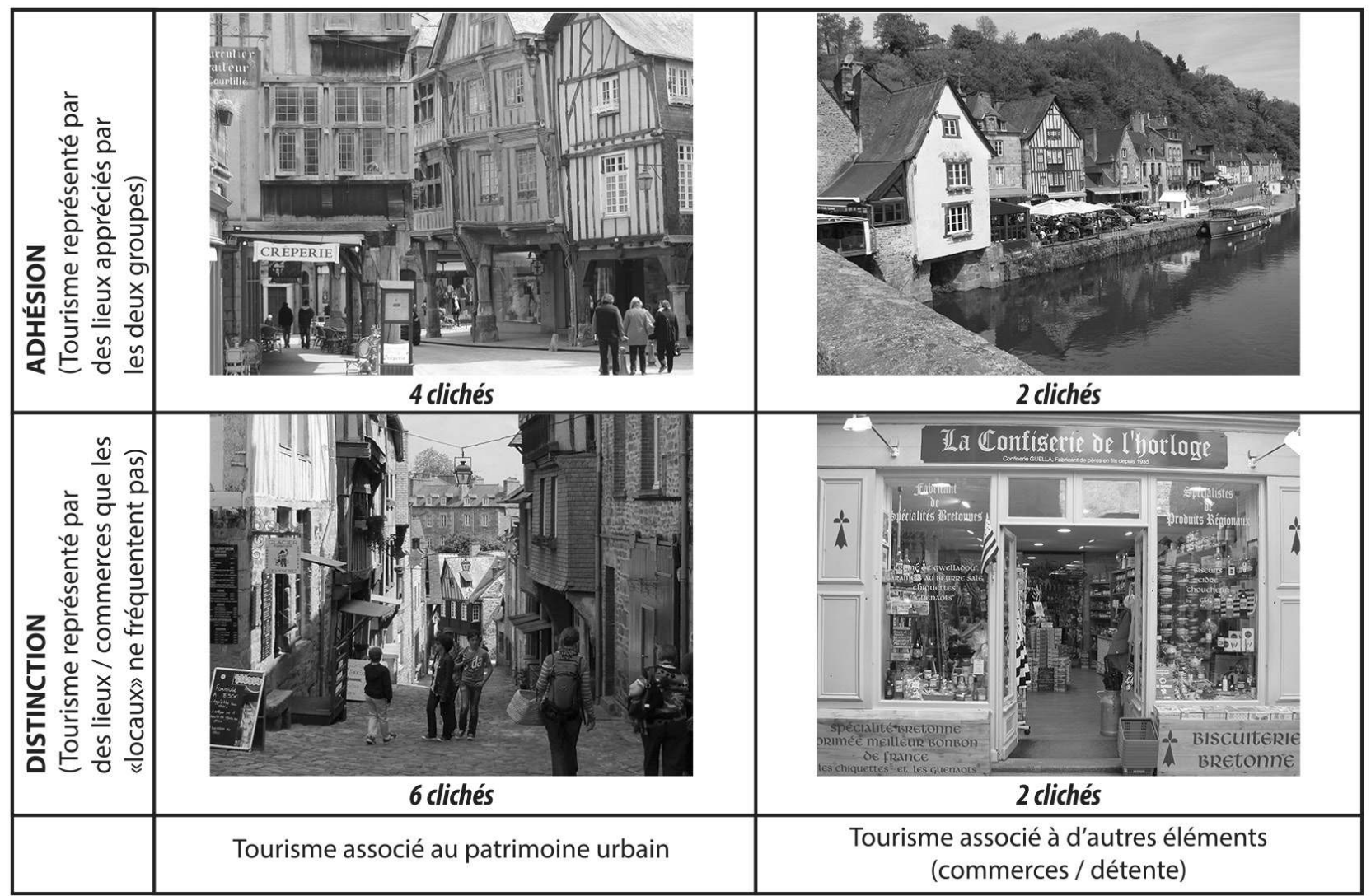

Figure 6 : Essai de typologie des représentations du tourisme par les résidents Try-outs on the Typology of the representations of tourism by inhabitants

quation avec les images promues dans les guides de voyage ou brochures touristiques, lorsqu'il est demandé aux résidents de conserver une seule photographie en souvenir de leur ville, on s'éloigne davantage de ces images. Deux facteurs semblent avoir un impact sur les « réponses photographiques » des participants : le lieu d'habitation et l'âge. On tend à s'éloigner davantage de l'image de destination chez les plus jeunes répondants (les 18-29 et les 30-39 ans). Également, les photographies étant toutes prises dans la ville-centre, le lieu d'habitation intervient davantage chez les répondants y habitant, leurs clichés étant quasi systématiquement pris à proximité du lieu de résidence.

Un premier groupe de répondants, ayant tous la quarantaine ou plus, a photographié des lieux centraux, largement promus par les organisations de mises en tourisme et en patrimoine. Néanmoins, si leur choix s'est porté sur les «incontournables" des guides de voyage, le lieu choisi est l'« incontournable » le plus près de chez soi. Il semble ainsi que cette question engendre une réponse photographique plus personnelle, mais qui est tout de même « cadrée » par les guides. La ville qui est représentée subit l'influence de la ville habitée, mais dans une mesure tout de même limitée. Cette corrélation entre le lieu de résidence et le sight choisi apparaît essentiellement chez les gens résidant dans le centre historique (intra-muros ou secteur sauvegardé) ou encore ceux de Lanvallay (commune voisine) ayant une vue sur le port et le rempart. Pour les résidents d'autres secteurs de la ville ou encore d'autres communes de la CODI (des mêmes groupes d'âge), l'image qu'ils souhaitent conserver est très liée à celles qui représentent le mieux la ville et le patrimoine. Ce sont donc trois questions qui donnent lieu à des « réponses photographiques » quasi identiques.

Un deuxième type de réponse apparaît : une photographie plus personnelle, essentiellement fournie par des résidents de moins de quarante ans. On retrouve chez ce deuxième groupe, des réponses 
qui se rapprochent davantage de celles obtenues par Bigando (2008) à Artigueloutan : "Pour moi ce serait la rue de la Chaux. C'est pas vraiment pour la rue en soit, mais parce que c'est là qu'on rencontre les amis. C'est plus ça qui serait important que des beaux bâtiments ou une jolie place. » (R07)

Pour cette résidente d'une trentaine d'années, née à Dinan, et y ayant passé la quasi-totalité de sa vie, l'image à conserver en souvenir de la ville est davantage liée aux liens sociaux, à l'ambiance, qu'à la ville en soit, ou à son patrimoine. Il en va de même pour deux autres jeunes résidents, natifs de Dinan. Les jeunes néo-Dinannais fournissent aussi ce type de réponses assez personnelles. Les deux premiers que nous avons rencontrés ont opté pour une prise de vue leur rappelant leur arrivée à Dinan.

"Ce serait la vue que j'ai en sortant de chez moi. [...] Avant d'acheter [...], tu te poses des questions, t'hésites, et puis je me rappelle, j'étais assis sur la p'tite marche là devant le bar, et je réfléchissais, [...] et en regardant cette vue, je me suis dis: "Ah non mais c'est quand même un super cadre!" " (R08. Un trentenaire ayant emménagé à Dinan après y avoir acheté un bar)

"La rue de la Mittrie. Je la trouve vraiment très jolie cette rue... Et puis surtout c'est là que j'ai habité quand je suis arrivée à Dinan! » (R10)

Vu le nombre restreint de personnes rencontrées, il est difficile de croiser les informations (classe d'âge, secteur de résidence, natif ou non, etc.). Néanmoins, au regard des éléments plus personnels que fait ressortir cette question, on perçoit davantage de distinctions selon ces différents critères que pour les autres questions. Il serait certainement intéressant de poursuivre l'expérience, avec un nombre plus important de résidents, afin de tenter de voir à quel point l'âge, ou le fait d'être natif de la ville ou non, par exemple, peut influencer les résultats.

\section{Conclusion}

Les villes historiques mises en tourisme et en patrimoine se doivent de répondre à de fortes exigences d'esthétisation. Dinan n'échappe pas à cette tendance. Alors que son image pittoresque de cité médiévale attire particulièrement les touristes, la ville se doit d'être à la hauteur. Emma Waterton (2010) souligne que les acteurs de ce double-processus de patrimonialisation et mise en tourisme, par la création et la gestion de l'« image patrimoniale », contrôlent non seulement ce patrimoine mais aussi la conception que se font les populations de ce qu'est le patrimoine, et de ce qu'est le passé, leur passé. Il semble bien que ce double processus entraîne une inévitable réduction, ne conjuguant plus mémoire(s) au pluriel mais au singulier. Un seul patrimoine, et par le fait même, une seule mémoire ne peut perdurer, celle permettant de servir les intérêts actuels. Cette situation n'est pas, bien entendu, sans conséquences. Parmi celleslà, nous pouvons noter une certaine convergence des regards. Alors que tout semble opposer touristes et résidents, étant au sein de la ville historique à l'étude, dans des espaces-temps complètement différents, ils posent un regard sur celle-ci qui tend à s'homogénéiser. Dans un second temps, nous pouvons également noter les prémisses de ce que Tom Mordue (2010) a identifié comme une forme d'aliénation. Deux éléments majeurs se dégagent de l'exemple dinannais :

- La mise en valeur du patrimoine, se concentrant depuis les dernières années, sur le patrimoine militaire, plus exactement, sur la mise en valeur du rempart. Ce patrimoine n'a dans ce cas, plus de valeur d'usage mais essentiellement une valeur d'image et est souhaité comme tel, comme le prouvent les démolitions de bâtiments à vocation résidentielle lui portant ombrage.

- Une certaine forme de rejet, de la part des résidents, des lieux considérés comme (trop) touristiques. La rue du Jerzual (et du Petit-fort) principalement, est exclue de l'espace de vie des Dinannais, considérée comme un lieu autre. Ce rejet pourraitil, à terme, s'étendre à l'ensemble du centre historique?

Toutefois, nous ne souhaitons pas prédire, à l'instar de J.-L. Roques (2009), la fin de la petite ville qu'est Dinan, ni sa mutation en une ville-musée. Un élément essentiel, apparu au fil de notre travail d'enquête est l'affection des touristes et l'attachement des Dinannais pour cette ville, «à taille humaine ${ }^{21} »$. Ainsi, nous croyons qu'elle peut (et doit) rester vivante, animée et habitée, et ce, autant par les habitants permanents que par ceux de passage, qui l'habitent temporairement. C'est certaine-

21. De tels propos ont été exprimés par de nombreux touristes et résidents rencontrés. 
ment la voie la plus sûre lui permettant de conserver sa mémoire, ou plutôt ses mémoires, des mémoires plurielles.

\section{Remerciement}

Bien que ce travail de recherche n'ait pas été réalisé dans le cadre de mon actuel travail de thèse, je tiens à remercier mon directeur, monsieur Philippe Duhamel, de même que Solène Gaudin, Benoît Montabone et les deux relecteurs de la revue pour leurs conseils et commentaires avisés.

\section{Bibliographie}

Bailly A., Baumont C., Huriot J.-M., Sallez A., 1995. Représenter la ville, Paris, Economica, 112 p.

Bigando E., 2006. La sensibilité au paysage ordinaire des habitants de la grande périphérie bordelaise (communes du Médoc et de la Basse Vallée de l'Isle), thèse de doctorat en géographie, Université de Bordeaux 3, 490 p.

Bigando E., 2008. Artigueloutan et ses paysages ordinaires. Le paysage en perspectives, version électronique, http://web. univ-pau.fr/ ftesson 1/tesson/images/RAPPORT_FINAL_ ARTIGELOUTAN_WEB.pdf, consulté le 27 mars 2013.

Bigando E., 2013. De l'usage de la photo-elicitation interview pour appréhender les paysages du quotidien : retour sur une méthode productrice d'une réflexivité habitante, Cybergeo : European Journal of Geography [En ligne], Politique, Culture, Représentations, document 645, mis en ligne le 17 mai 2013, URL: [http://cybergeo.revues.org/25919; DOI : 10.4000/cybergeo.25919].

Boorstin D. J., 1963 [1961]. L'Image. Ou ce qu'il advint du rêve américain [The Image: A Guide to Pseudo-Events in America], Paris, R. Julliard, $328 \mathrm{p}$.

Bourdieu P., Boltanski L., Castel R., Chamboredon J.-C., 1965. Un art moyen. Essai sur les usages sociaux de la photographie, Paris, Les éditions de minuit, 360 p.

Cazes G., 1998. Le renouveau du tourisme urbain. Problématiques de recherche, in Cazes G., M, Potien F., Le tourisme et la ville: Expériences européennes, Paris, L'Harmattan, p. 13-26.

Chazaud P., 1994. Quels espaces, quels loisirs, quelles stratégies pour le tourisme urbain d'agrément?, Cahiers Espaces, $n^{\circ} 39$, p. 44-52.

Chony F., 1992. L'allégorie du patrimoine, Paris, Seuil, 272 p.

ÉQuipe MIT, 2002. Tourismes 1 : Lieux communs, Paris, Belin, $320 \mathrm{p}$.

Gravari-Barbas M., 1997. Gestion-valorisation du patrimoine historique bâti et tourisme urbain : le cas d'Angers, in Commerçon N., Goujon P., Villes moyennes espace, société, patrimoine [Colloque, Mâcon, 1995], Lyon, Presses universitaires de Lyon, p. 397-410.

Gravari-Barbas M, 2001. La leçon de Las Vegas : le tourisme dans la ville festive, Géocarrefour, Vol. 76, n 2, p. 159-165.

Hatt E., Deletraz G., Clarimont S., Vlès V., 2011 . Retour sur images, EspacesTemps.net, 12.10.2011, [http://www. espacestemps.net/articles/retour-sur-images/].

InvoAs-Dantec D. (2003), «L'intérêt d'une mise en réseau : les villes et Pays d'Art et d'Histoire », in Cuillier F. (dir.), Les débats sur la ville. 5. Patrimoine et développement des cours de ville. $4^{e}$ Assises du Patrimoine du Grand-Ouest, Mayenne, Confluence, p. 147-157.

Lazzarotti O., 2000. Patrimoine et tourisme : un couple de la mondialisation, Mappemonde, $\mathrm{n}^{\circ}$ 1, p. 12-16.

Lazzarotti O., 2003. Tourisme et géographie : le grand dérangement, in Sтоск M. et al., Le tourisme : acteurs, lieux et enjeux, Paris, Belin, p. 255-277.

Lazzarotti O., 2011 . Patrimoine et tourisme: histoire, lieux, acteurs, enjeux, Paris, Belin, 302 p.

Lelli L., 2000. Le paysage ordinaire : l'exemple du Nord-Comminges (Haute-Garonne, France). Essai méthodologique et pratique, thèse de doctorat en géographie et aménagement, Université de Toulouse - Le Mirail, 325 p.

Leniaud J.-M., 1992. L'utopie française. Essai sur le patrimoine, Paris, Mengès, $180 \mathrm{p}$.

LÉVy J., Lussault M., 2003. Dictionnaire de la géographie et de l'espace des sociétés, Paris, Belin, 1033 p.

LuginbüHL Y., 1989. Au-delà des clichés. La photographie du paysage au service de l'analyse, Strates, n ${ }^{\circ}$, p. 11-16.

LuginbüHL Y., 2012. La mise en scène du monde. Construction du paysage européen, Paris, CNRS Éditions, $432 \mathrm{p}$.

Lynch K., 1998 [1969]. L'image de la cité [The image of the city], Paris, Dunod, 222 p.

Maccannell D., 1976. The Tourist. A new theory of the leisure class, New York, Schocken Books, 214 p.

Michelin Y., 1998. Des appareils-photos jetables au service d'un projet de développement : représentations paysagères et stratégies des acteurs locaux de la montagne thiernoise, Cybergéo, $\mathrm{n}^{\circ}$ 65, $13 \mathrm{p}$.

Miossec J.-M., 1977. L'image touristique comme introduction à la géographie du tourisme, Annales de Géographie, vol.86, $\mathrm{n}^{\circ} 473$, p. 55-70.

Mordue T., 2010. Time Machines and Space Craft : Navigating the Spaces of Heritage Tourism Performance, in Waterton E., Watson S., Culture, Heritage and Representation. Perspectives on visuality and the past, Farnham, Burlington, Ashgate, p. 173-194.

Robinson M., Picard D., 2009. The framed world: tourism, tourists and photography, Farnham, Ashgate, 263 p.

Roques J.-L., 2009. La fin des petites villes. Une modernité envahissante, Paris, L'Harmattan, $221 \mathrm{p}$.

Roussillon A., 2010. À propos de quelques paradoxes de l'appropriation identitaire du patrimoine, in CATtedra R., Garret P., Miller C., Volait M. (dir.), Patrimoines en situation. Constructions et usages en différents contextes urbains, Beyrouth / Rabat, Presses de l'Ifpo/Centre Jacques- 
Berque, [http://books.openedition.org/ifpo/908], consulté le 30 décembre 2013.

Russo A. P., 1999. Venise : la fin du «tout-tourisme », Le Courrier de l'Unesco, juillet/août 1999, p. 42-43.

Ryan C., 2002. Tourism and cultural proximity. Examples from New Zealand, in Annals of Tourism Research, vol. 29, $\mathrm{n}^{\circ} 4$, p. 952-971.

Tucker H., Emge A., 2010. Managing a World Heritage Site : the case of Cappadocia, in Anatolia : An International of Tourism and Hospitality Research, vol. 21, n 1, p. 41-54.

Urbain J.-D., 1993. L'idiot du voyage. Histoire de touristes, Paris, Payot, $270 \mathrm{p}$.

Unry J., 1990. The Tourist Gaze. Leisure and Travel Contemporary Societies, London, Sage, $176 \mathrm{p}$.
Vlès V., Berdoulay V., Clarimont S., 2005. Espaces publics et mise en scène de la ville touristique, Rapport final de recherche, Ministère délégué au Tourisme, Université de Pau et des Pays de l'Adour, Laboratoire SET, UMR 5603 CNRS-UPPA, version électronique, [http://web.univ-pau. fr/RECHERCHE/SET/Auteurs/Vles/EPT_CNRS5603.pdf], consulté le 5 octobre 2013.

Waterton E., 2010. Branding the Past : The Visual Imagery of England's Heritage, in Waterton E., Watson S., Culture, Heritage and Representation. Perspectives on visuality and the past, Farnham, Burlington, Ashgate, p. 155-172. 